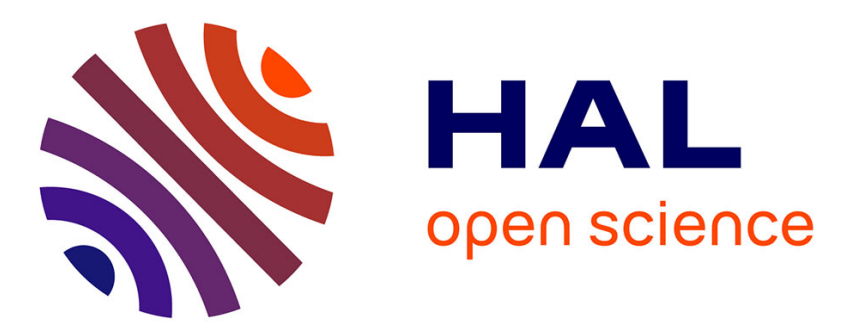

\title{
Explicit Knowledge and the Deliberative Layer: Lessons Learned
}

\author{
Séverin Lemaignan, Rachid Alami
}

\section{To cite this version:}

Séverin Lemaignan, Rachid Alami. Explicit Knowledge and the Deliberative Layer: Lessons Learned. IEEE/RSJ International Conference on Intelligent Robots and Systems (IROS), Nov 2013, Tokyo, Japan. hal-01979215

\section{HAL Id: hal-01979215 https://hal.laas.fr/hal-01979215}

Submitted on 12 Jan 2019

HAL is a multi-disciplinary open access archive for the deposit and dissemination of scientific research documents, whether they are published or not. The documents may come from teaching and research institutions in France or abroad, or from public or private research centers.
L'archive ouverte pluridisciplinaire HAL, est destinée au dépôt et à la diffusion de documents scientifiques de niveau recherche, publiés ou non, émanant des établissements d'enseignement et de recherche français ou étrangers, des laboratoires publics ou privés. 


\title{
Explicit Knowledge and the Deliberative Layer: Lessons Learned
}

\author{
Séverin Lemaignan and Rachid Alami \\ CNRS, LAAS, 7 avenue du Colonel Roche, F-31400 Toulouse, France \\ Univ de Toulouse, LAAS, F-31400 Toulouse, France \\ severin.lemaignan@laas.fr, rachid.alami@laas.fr
}

\begin{abstract}
Over the last four years, we have been slowly ramping up explicit knowledge representation and manipulation in the deliberative and executive layers of our robots. Ranging from situation assessment to symbolic task planning, from verbal interaction to event-driven execution control, we have built up a knowledge-oriented architecture which is now used on a daily basis on our robots.

This article presents our design choices, the articulations between the diverse deliberative components of the robot, and the strengths and weaknesses of this approach. We show that explicit knowledge management is not only a convenient tool from the software engineering point of view, but also pushes for a different, more semantic way to address the decision-making issue in autonomous robots.
\end{abstract}

\section{A KNOWLEDGE-ORIEnted ARChitecture}

\section{A. Towards the cognitive robot at LAAS}

Natural interaction and cooperation are on the (dare we say, short-term) agenda for the human-robot interaction community. They are keys to the broad class of interactive manipulation problems: several agents agree on a (more or less implicit) joint goal that requires some sort of cooperation to be successfully achieved. This class of problems involves both dialogue and manipulation and they are often initially underspecified: they require iterative and interactive resolution.

Over the last years we have focused our efforts on identifying the cognitive prerequisites of these challenges, and giving them experimental reality on the robots: what is required for sentences like "Let's set the table together" to be understood by the robot, correctly interpreted in the spatial and temporal context of the interaction, and eventually transformed into a set of actions.

We have chosen to tackle the challenge from several ends: human-aware navigation and motion planning [1], situation assessment coupled with motion planning [2], projection of "mightabilities" that anticipates what surrounding agents may do [3], and the design and deployment of a pervasive knowledge-oriented software architecture.

The last item is the focus of this paper: what "knowledgeoriented" architecture means, and what its benefits and drawbacks are.

\section{B. Scope of the article}

In essence, this article portrays our use of explicit semantic interfaces to integrate several deliberative components into a decisional architecture for our robots. It reports on our experience with acquiring, managing and reusing grounded knowledge in the context of human-robot interaction.

Unlike previous publications by the authors that introduced the deliberative modules of our robots independently from each other, this contribution is an account of the importance of explicit knowledge manipulation when integrating them into a large cognitive architecture.

While we do not present experiments in this paper, the ideas and techniques we present all have been implemented and tested on several robots (see previously reported experiments in [4]-[7], [9]). Videos presenting some of these experimental results can be watched online at http://www. laas. fr/ $\sim$ slemaign.

Also note that this paper does not provide extensive review of existing literature. We kindly refer the reader to the aforementioned articles for in-depth discussion of related works in the different fields of cognitive architectures for robots.

\section{Article overview}

In the next section, we first introduce the deliberative layer of our robots at the functional level. It represents what we call a knowledge-oriented architecture, built around a central knowledge management tool called the OpenRobot Ontology (ORO) server.

The following sections present each of the facets of this deliberative layer: how knowledge is produced asynchronously from geometric reasoning, how the robot conducts grounded multi-modal interaction with humans, how knowledge is used by decision-making components like the robot controller or the task planner, and finally, how our knowledge-oriented architecture enables the implementation of specific internal cognitive processes.

We conclude the article with a discussion about the strengths and weaknesses of such a knowledge-oriented architecture, from three perspectives: the software architect, the AI expert and the cognitician.

\section{THE LAAS DELIBERATIVE LAYER}

Fig. 1 gives an overview of the connections between the deliberative components of our architecture [8].

This architecture moves away from standard layered approaches. Interactions between components at the deliberative level are mostly bidirectional and we do not introduce layers 


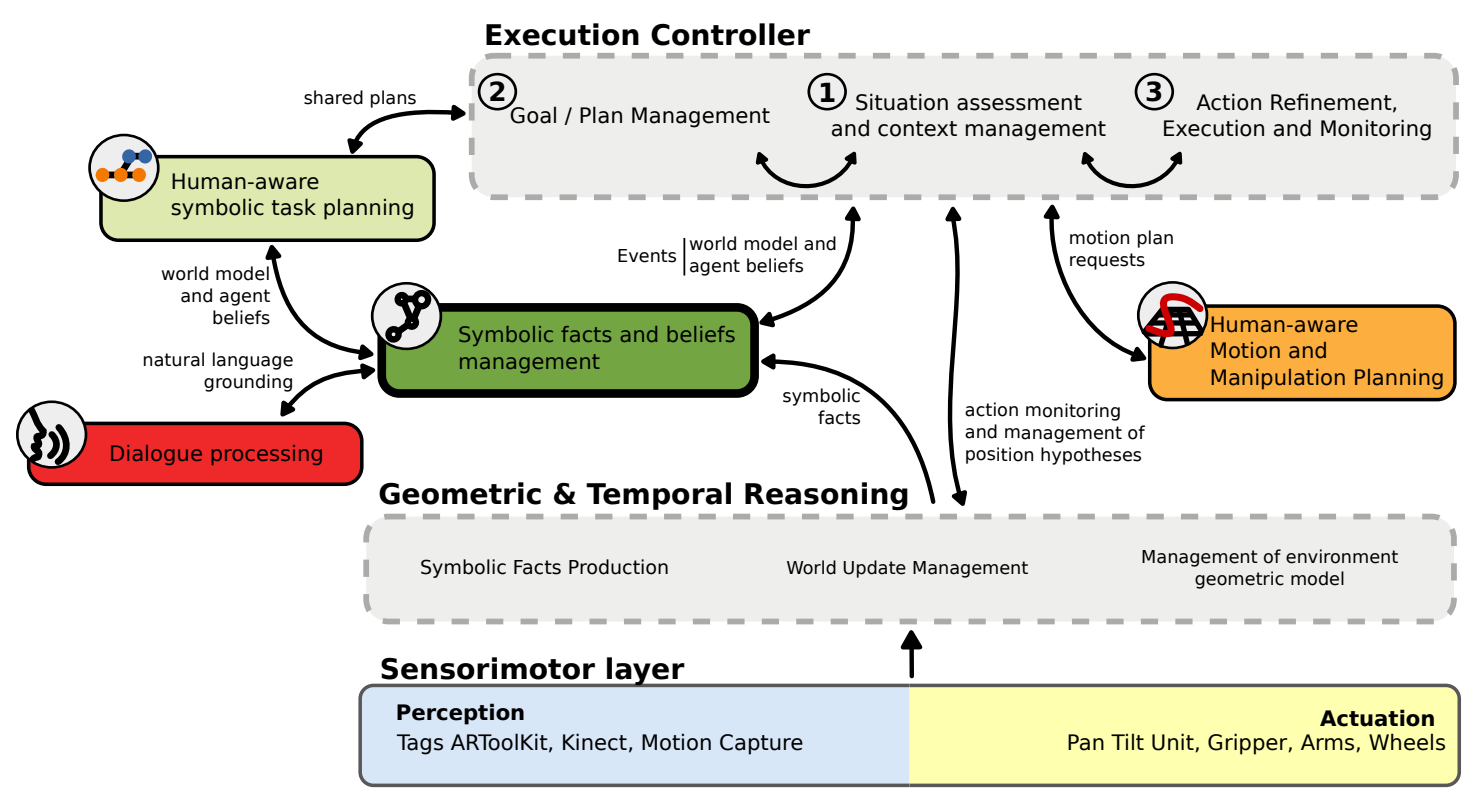

Fig. 1. Overview of the LAAS deliberative layer. Knowledge is centrally managed in an active semantic blackboard, pictured above with a thick border.

of abstraction amongst software components ${ }^{1}$. This is nicely illustrated by the dialogue input processing. This component does not simply act as an alternative perceptual input to the symbolic database; it also actively queries previously acquired knowledge to disambiguate and validate the newly created symbolic knowledge (see section IV).

Our architecture relates to Beliefs, Desires, Intentions (BDI) architectures. BDI architectures are primarily focused on practical reasoning, i.e. the process of deciding, step by step, which action to perform to reach a goal (as summarized by Woolridge [11]). The management of the interaction between knowledge (the beliefs) and task and plan representation and execution (the desires and the intentions) is central, and aims at selecting at each step the best subgoal. It becomes then an intention that the robot commits to.

This interaction between knowledge and actions is also central to our approach (as for any cognitive system): it is one of the activities of the robot, actually shared between communication components (that can acquire desires from interaction with agents, amongst other things) and an execution controller that may decide to take an incoming desire into account to create its own internal goals. The controller generates and manages intentions from these goals with the help of a symbolic task planner, that also has direct access to the knowledge base.

This activity is however not the backbone of our architecture. Other activities are conducted in parallel, without being explicitly considered as desires: assessment of the situation and the environment, dialogue (including performative dialogue that can possibly change the internal state of the robot, but does not lead to the creation of desires, like question answering or statement assertion), various background monitoring and recognition tasks, etc.

\footnotetext{
${ }^{1}$ We do have lower-level modules to execute actions or manage sensors, but all cognition-related modules reside at the same level.
}

Regarding the anchoring question, this architecture is bidirectional. The components we described provide a bottomup grounding process: geometric reasoning and dialogue processing modules constantly build and push new symbolic contents about the world to the knowledge base where it becomes accessible to decisional layers. In parallel, the knowledge base relies on reasoning in a top-down way to produce new facts that may in return trigger physical behaviours.

\section{Knowledge model}

In our architecture (Fig. 1), knowledge manipulation relies on a semantic blackboard: a central server (the ORO server [4]) stores knowledge as it is produced by each of the deliberative components. It conversely exposes a j son-based RPC API to query the knowledge base [12].

Knowledge is represented as RDF triples in the OWL sublanguage. Each time triples are added or removed from the knowledge base, a Description Logics reasoner (PELLET ${ }^{2}$ ) classifies the whole ontology and inserts all possible inferred triples.

Relying on RDF triples and Description Logics has advantages such as the availability of numerous mature opensource libraries to manipulate the ontology, interoperability with several major on-line knowledge bases (like OPENCYC, WORDNET or DBPEDIA), open-world reasoning, and the formal guarantee of decidability (it is always possible to classify a Description Logics ontology).

It also has notable limitations, both fundamental (the suitability of Description Logics when reasoning on -typically non-monotonic- commonsense knowledge is questionable) and practical: RDF triples imply only binary predicates ( subject predicate object $\rangle$ ), which constrains the expressiveness of the system or leads to cumbersome reifications.

\footnotetext{
${ }^{2}$ http://clarkparsia.com/pellet/
} 


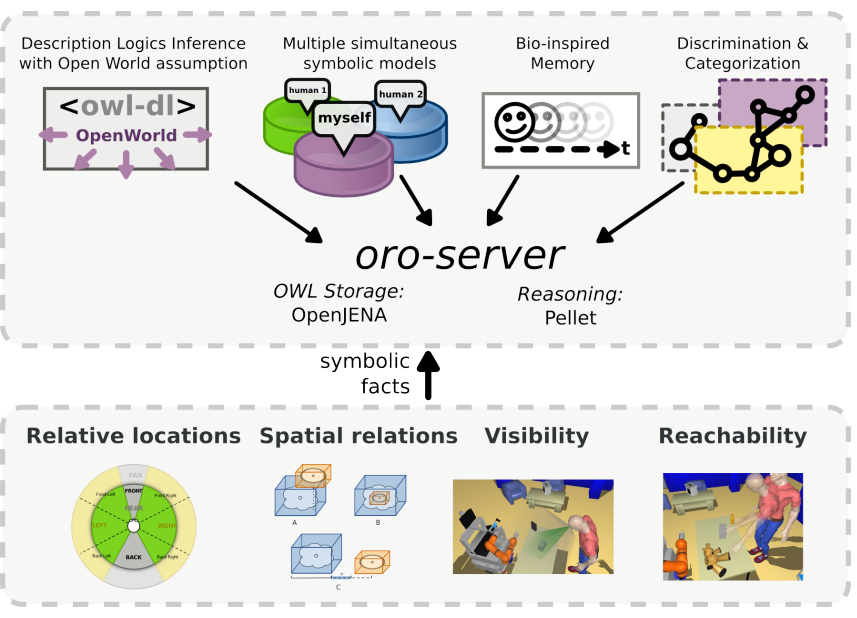

Fig. 2. Functional overview of knowledge base (ORO server, top part) and the geometric situation assessment module (SPARK, bottom part)

Alternatives exist (like KNOwRoB [13]) that mix RDF with more expressive logic languages like PROLOG, at the price, however, of other limitations, like closed-world reasoning or immutable T-Box. The classification performance is another issue: from our experience, with an ontology sized for a standard experiment (about 100 classes and 200 instances), classification typically takes about $100 \mathrm{~ms}$, which becomes problematic during interactions. Besides, the performances are difficult to predict, since a seemingly inoffensive new statement may indirectly change radically the logical complexity of the whole knowledge model and lead to notable degradation of classification time.

This knowledge model also largely excludes representation of continuous phenomena (like time) or uncertain phenomena. When required (for instance for action recognition), these are managed inside the corresponding components, and are not exposed at the semantic level.

Tools to manipulate and reason over ontologies are readily available, mature, and already well accepted in the robotic community [13], [14]. While alternatives like Answer Set Programming have also been successfully investigated in robotics [15], [16], in particular to deal with non-monotonic reasoning, we did not actually hit any brick wall while working with OWL ontologies. We may reconsider this choice at a later stage, but until now it has proven an effective framework to quickly explore implementations of new cognitive abilities (for instance, it has been conceptually and technically easy to add support for independent knowledge models, one per the agents the robot interacts with -see section VI-A).

Besides, because ontologies and RDF statements are relatively simple concepts to grasp, it also effectively helped to grow awareness amongst colleagues on the significance of the "semantic level" when developing new components for the robot.

\section{Situation Assessment}

Anchoring perceptions in a symbolic model requires perception abilities and their symbolic interpretation. We rely on a dedicated geometric and temporal reasoning module called SPARK (SPAtial Reasoning \& Knowledge [9]). It is a situation assessment reasoner that generates symbolic knowledge from the geometry of the environment with respect to relations between objects, robots and humans (Fig. 2), also taking into account the different perspective that each agent has on the environment.

SPARK is an amodal geometric model of the environment that serves both as basis for the fusion of the perception modalities and as bridge with the symbolic layer. This geometric model is continuously updated at run-time by the robot based on its sensors (in our experiments, objects are identified and localised through 2D barcodes, while humans are tracked with Kinect-like devices, optionally assisted by motion capture to accurately track the head motion, which is required to compute what the human is looking at).

Computed symbolic relations: Over twenty physical relations are continuously computed by SPARK: spatial relations, both agent-independent (like ison, isNext To or isMoving), and agent-dependent (like isNear, leftTo), and also affordances: sees, looksAt, pointsAt, canReach. Visibility (with two levels corresponding to the field of view and the field of attention) and pointing are computed by placing virtual cameras in the SPARK 3D model, while reachability relies on inverse kinematics. Several filters (based on hysteresis, expected object behaviour, etc.) allow to partially account for perception noise [7].

Ego-centric and allo-centric frames: SPARK enables perspective-taking: spatial relations between entities can be computed from different viewpoints, which let the robot build a different, perspective-aware symbolic model of the environment for each agent it interacts with. These models are separately stored in the knowledge base.

This allows us to deal with ambiguities that arise when one speaker refers to an object within a reference system (or changes the reference system, i.e. switches perspective) without making the reference frame explicit [17], [18].

As a result, the robot stores models of the environment either in the ego-centric reference frame (from the robot perspective) or in the allo-centric frame (addressee-centred).

\section{COMMUNiCATION}

Natural language grounding: Natural language processing is one of the fields of human-robot interaction for which the introduction of the semantic layer has been most beneficial. In [6] we detail the techniques and the tool called dialogs that we have developed for natural English language parsing and grounding, along with verbalisation and (minimalist) dialogue management.

Natural language input (in experiments, we rely on an Android-based interface, with Google speech recognition) is parsed into a grammatical structure, and atoms of each sentence are resolved with the help of the ontology to ground concepts like objects (i.e. when a user says "pick the can", resolve to which instance of can the user is referring to) and actions. Sentences are sorted into questions, desires and statements, and processed accordingly. 
The system supports quantification ("give me $\{\mathrm{a} \mid$ the I some I all I any I ... $\}$ can"), thematic roles (action-specific predicates that qualify the actions), interactive disambiguation (the robot asks questions when it needs more information), anaphora resolution ("give it to me") based on dialogue history. It also permits knowledge extension by learning new semantic structures (for instance, a sentence like "learn that cats are animals" is converted into < Cat subclassof Animal), interprets common temporal and place adverbs (like above or tomorrow) and translates to a certain extend states ("I'm tired") into experiences ( $\langle$ HUMAN experiences state_1, state_1 hasFeature tired $\rangle)$.

Limits of disambiguation at semantic level: One prototypical example of semantic disambiguation has been given in [5] with the child game spygame: two players are facing each other with a set of random objects in-between, one player mentally choose one object, and the other player has to guess the object by asking closed questions like Is your object small or large? Based on the knowledge it has acquired, the robot is able to minimize the number of questions required to find the object.

When playing this kind of game, however, the issue arises that the robot has no way to select which knowledge about the object is relevant in the interaction context. For instance, the knowledge base may store facts like $\langle$ obj1 type ActiveConcept) (which internally means that this concept was mentioned in a discussion in the last few seconds): this information is not a relevant property of obj1 when trying to disambiguate concepts with humans. This distinction between internal knowledge (meaningful to the system only) and common knowledge (whose meaning is understood by all the interactors) has not been properly dealt with in our architecture.

Besides, even knowledge that belongs to the common knowledge may not be appropriate in a given interaction context. For instance, the system may compute that at a given instant the human is looking at the object: < human looksAt obj1). This property makes sense to both parties, but in the context of the spygame, we would like to mainly use immanent properties, not volatile like a gaze. More research is required to identify relevant interaction contexts and knowledge classes attached to them.

\section{A. Multi-modal communication}

Because all components rely on the same RDF formalism to format their outputs, the different communication modalities (explicit like verbal, deictic or based on gaze, or implicit like postures) are presented in a homogeneous way. The dialogue grounding process makes use of them at two distinct levels.

First, particular steps of the grounding process explicitly check for the presence and value of specific facts: for instance, when a set of instances matches a category (the human says "give me the bottle" and the robot knows about three bottles), the module may decide (it actually depends on the quantifier preceding the class) to discard some of them based on their visibility for the speaker (implicit communication context built on the human posture).
Another example, when the human says "this", the robot checks if the human is currently pointing at some object. In that case, this is replaced by the object focused on.

Note that, while the system benefits from the complementary modalities, they are not all required. The dialogue system would for instance happily run with only the verbal modality, at the cost of weaker interaction.

The second level of integration of multi-modality is implicit: by computing symbolic properties from the geometry, richer descriptions and hence discrimination possibilities are available: for instance, if reachability is available, the robot may ask "do you mean the bottle that is accessible to me?" to discriminate between the three bottles. That way, procedures relying on discrimination transparently benefit from added modalities.

\section{ROBOT CONTROL}

\section{A. Desires and experiences}

Our robot execution controllers (OpenPRS-based SHARY or Python-based PYRoBOTs) have deep integration with the knowledge base. It serves as the primary source of information for semantic-aware decision-making.

We split the interaction situations stemming from the situation assessment and communication components in two categories: desires (performative act) and experiences (assertive act).

Desires are typically human orders ("Give me that book"). The nature of the desired action (to pick, give, look, bring, show...), along with the action parametrization (what is acted on? who should perform the action? etc.) are extracted from the knowledge base, and either passed to a task planner (presented in the next section) or executed if the procedure is directly available.

Experiences, on the other hand, comprise of emotions, states and questions (when asking a question, we consider the human to be in an interrogative state). When the knowledge base recognizes that an agent experiences a particular emotion or state, the execution controller may decide to handle it, typically by trying to answer the question or using the emotional or physical state as a parameter for subsequent actions. As an example, when the speaker says "I feel tired", we change the motion planner parametrization to lower the effort the human needs to provide for the following joint manipulation tasks. Note that this example has been implemented as a proof-of-concept. We have not yet tried to define a theoretical framework that would support action alteration based on the user's experienced states.

\section{B. Event-driven control}

The ORO server proposes two paradigms to access its content: RPC-style queries (based on the standard SPARQL language) or events. In its simplest form, a module can subscribe to an event by passing through an event pattern (in its basic form, a partial statement like ( ? type Elephant〉) and a callback. Each time a new instance of elephant appears in the knowledge base, the callback is triggered. 
This allows us to write reactive robot controllers with a high level of expressiveness: for instance, by subscribing to the event/human1 desires ?action, ?action type Look, ?action hasGoal myself $\rangle$, we could trigger a behaviour when the human expresses (through dialogue, gestures...) that he wants to look at the robot itself.

The robot controller designer does not need to directly care about how this desire is produced (this is delegated to perception modules), he can focus on the semantic of the desire.

Note also that we take advantage of the reasoning capabilities of the system: for example, the goal of the action ( $\langle$ action hasGoal myself $\rangle$ ) may not be explicitly asserted, but inferred by the reasoner based on other assertions.

\section{Task planning}

Our execution controllers rely on symbolic task planning to convert faraway desires into a succession of atomic actions. We use in our architecture the HATP planner (for Human Aware Task Planner [10]). HATP is based on a Hierarchical Task Network (HTN) refinement, which performs an iterative task decomposition into sub-tasks until reaching atomic actions. The planning domain defines the set of methods describing how to decompose a task and represents the procedural knowledge of the robot.

In order to produce a collaborative behaviour, the robot plans not only for itself but also for the other agents. The planning domain of each agent is instantiated from the agentspecific model in the ORO server. The resulting plan, called shared plan is a set of action streams, one per agent involved in the goal achievement. HATP can also be tuned by setting up different costs depending on the actions to apply and by taking into account a set of constraints called social rules. This tuning aims at adapting the robot's behaviour according to the desired level of cooperation of the robot.

Two important remarks: because HATP is a generic symbolic task planner, we have been able to design a planning domain at a semantic level which is close to the one used in the human-robot dialogue (the planner vocabulary contains concepts like give, table, is on...). Hence only a few ontology rules have been required to map both the knowledge extracted from the situation assessment and the statements originated from the verbal interaction to the planner domain.

Second remark, after some research (see appendix B of [19] for a detailed discussion) we have decided to represent neither the planning domain nor the resulting plans in the knowledge base: the planning domain (with task pre- and postconditions) is stored in a specific format, outside of the central declarative knowledge repository, and the plans are directly communicated to the robot controller. Thus, like many other cognitive architectures, we have independent declarative and procedural knowledge stores.

\section{INTERNAL COGNITIVE PROCESSES}

\section{A. Theory of Mind}

Theory of Mind (originally defined in [20]) is the cognitive ability that a subject possesses to represent the mental state of another agent, possibly including knowledge that contradicts the subject's own model: for example, a book can be at the same time visible for myself, and not visible for you.

Children develop this skill, which is essential to understand others' perspectives during interactions, around the age of three. It supposes the ability to build, store and retrieve separate models of the knowledge of the interactors.

Our knowledge base implements such a mechanism: when the robots infers a new agent has been introduced in the knowledge base, it initializes a new, independent, ontology for this agent. All the ontologies that are created share the same common-sense knowledge, but rely on each agent's perspective for the actual instantiation: the robot (geometrically) computes that the book is in its own field of view, but not in the human one. The robot knowledge contains the fact 〈 book isvisible true while the human model contains < book isvisible false .

One classical application of this cognitive skill is the socalled False-Belief experiment (also known as the Sally and Ann experiment) [21]: a child is asked to watch a scene where two people, A and B, manipulate objects. Then A leaves and $\mathrm{B}$ hides away one object. When A comes back, we ask the child "where do you think A will look for the object?". Before acquiring a theory of mind, children are not able to separate their own (true) model of the world (where they know that the object was hidden) from the model of A, which contains false beliefs on the world (A still thinks the object is at its original position since he did not see B hiding it). Using separate knowledge models in the knowledge base, we have been able to replicate this experience with our robots [7].

\section{B. Working memory}

The ORO server also features a mechanism to mimic minimalistic forms of biological memory. When new statements are inserted in the knowledge base, a memory profile is optionally attached to them.

Three such profiles are predefined: short term, episodic and long term. They each correspond to a different lifetime for the statements (respectively 10 seconds, 5 minutes and no time limit). After this duration, the statements are automatically removed from the knowledge base.

This approach is limited. In particular, episodic memory primarily refers to the semantics of the statements (that is expected to be related to an event) and not to a specific life duration.

We rely however on this short term memory for a particular use-case: active concepts. Some modules, like the natural language processor, use the short term memory profile to mark for a few seconds important concepts that are currently manipulated by the robot. For example, if a human asks the robot: "Give me all red objects", the human, the Give action, and each red objects that are found are successively marked as active concepts by inserting statements such as $<$ human type Activeconcept) in the short-term memory (which can be considered, in this case, to be a working memory). We use this feature to trace certain knowledge-related processes. 


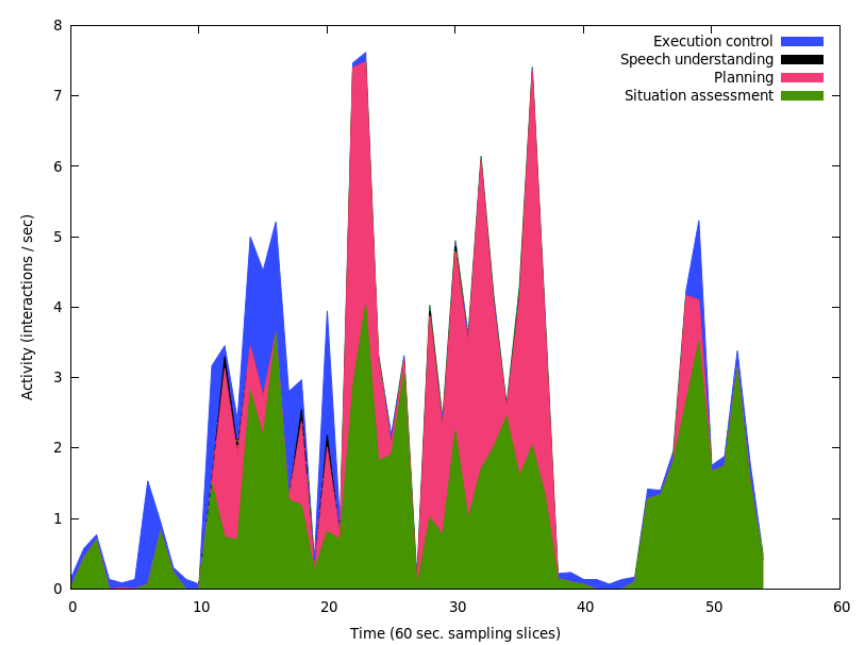

Fig. 3. This diagram shows the average number of interactions with the knowledge base during a one-hour long experiment with the robot. Interactions are either knowledge alteration (addition/removal of statements) or queries. They are averaged on a sliding window of 60 seconds.

\section{Cognitive activity}

Fig. 3 is a plot of the interactions (knowledge alterations or knowledge queries) between the robot's modules and the central knowledge base during a one-hour long experiment. Because most of the communication between the modules are in fact supported by the knowledge base in our architecture (Fig. 1), it reflects well the exchanges of semantic informations within the deliberative layer of the robot, which we can tentatively call the cognitive activity of the robot.

This simplistic measurement needs to be refined to take into account the fact that the raw number of RPC calls to the knowledge base only imperfectly reflect the actual cognitive processes running on, but it opens interesting perspectives nevertheless. We could for instance investigate possible correlations with the cognitive activity of a human partner (measured via EEG or qualitative feedback).

\section{DISCUSSION}

Altogether, the components we have presented compose an architecture that we call knowledge-oriented:

- Knowledge is explicitly stored in one central and consistent repository of facts, accessible for all modules.

- Knowledge is represented in a strict formalism (OWL statements) and with a clearly defined vocabulary (stated in the common-sense ontology).

- The first two points enable a loosely-coupled architecture where modules can be removed or replaced easily by other ones as long as they share the same semantics (modules are defined by the knowledge they produce),

- We adopt a symbolic, reactive, event-driven approach to robot control. By managing events at the same level as the reasoner, we take full advantage of the inference abilities of ORO to trigger events whose true conditions can be inferred.

- Finally, this architecture allows for the combination of very different knowledge modalities in a single homogeneous environment, bringing mutual benefits to components. For instance, the dialogue processing module can perfectly run without any geometric perception, but its disambiguation routines can transparently benefit from it when available (since richer symbolic descriptions of objects are then available).

Those items are not new per se. It seems however interesting to underline the shift of focus this implies during the design and integration phases. We can adopt three perspectives to discuss the techniques we have presented here: first, the architect perspective: what are the pros and cons of our approach from an engineer's point of view. Then, the logician perspective: does the promises of automated reasoning for robots really come to fruition with ontologies? And finally the cognitician perspective or how explicit knowledge manipulation endows our robots with new cognitive capabilities.

\section{A. The Architect view: loose coupling and modalities merging}

In this article, we have presented several sub-systems of our robots: a module for geometric reasoning and situation assessment (written in $\mathrm{C}++$ ), a natural language interface (written in Python), a symbolic task planner (written in $\mathrm{C}++$ ), two execution controller (one written in Python, one in PRS). These modules communicate together through RDF statements with only a few exceptions.

Since these statements are anchored in the robot's pool of knowledge, they actually convey shared and unambiguous meaning through the system. This has one important consequence from the perspective of the system architecture: interfaces between modules are only defined in terms of knowledge consumption and/or production. This brings good decoupling properties. For instance, the output of the dialog module is a new situation desired by the speaker. This module can be transparently replaced or completed by any other module that produces the same chunks of knowledge. Only a thin protocol layer remains to get the modules to interact with each other. This allows us to implement (otherwise difficult) merging of radically different perception modalities like vision (the robot sees an object on a table: the output would look like $\langle o b j 1$ ison table1 $\rangle$ ) and verbal description (a human says to the robot the object on the table is red: it leads to a query < ? ison table1〉 and then a new assertion: 〈 obj1 hascolor red).

Another consequence is that the communication channels are de facto defined by the semantics of the information they convey. This allows the system designers to think in terms of which module produces or needs which knowledge, instead of which module produces which service. We found this approach to be helpful when implementing cognitive functions like the theory of mind, the perspective taking or the natural language grounding.

Finally, another good property of knowledge-based communication between modules is the cognitive observability of the system: by logging queries between modules (which is even easier in our case since we rely on a central knowledge management), one can trace the interactions between the 
robot subsystems at a high abstraction level. We address the significance of this property from the cognitive point of view below.

The knowledge-oriented architecture also has shortcomings. We can name here a few.

Because the sources of knowledge the robot has to deal with are multiple and often not known at startup (typically, the knowledge generated by a verbal interaction with a human), it is difficult to formally guarantee reliability.

Also, it must be noted that actuation itself is directly piloted by the execution controller through standard RPC calls (ROS actions and/or Pocolibs requests): no knowledge abstraction takes place.

Also on the sensing side, we have to bypass the semantic layer in certain cases (extension of the symbolic task planner with geometric constraints is one example).

Many aspects like uncertainty management, modal logics, non-monotonic reasoning are not well addressed with the Description Logic formalism, and remain to be explored to gain the level of expressiveness required to cover many of the more complex human-robot interaction scenarios.

\section{B. The Logician view: the importance of trivial inferences}

Where to find milk? Milk is a subclass of dairy which is itself a subclass of a perishable goods. The usual storage place for perishable goods is the fridge, so the milk is likely to be found in a fridge.

This example of reasoning, quoted from Moritz Tenorth, is a good example of simple yet non-trivial reasoning. As a matter of fact, only very few of such prototypical reasoning cases were positively identified in our scenarios and experiments (and consequently implemented as rules in ORO).

The design choices of our architecture partially explain that fact: first, the planning task (which is a typical reasoning task) is delegated to a dedicated, external planner (HATP). Then, time is not represented in ORO, and consequently no temporal reasoning takes place at this level: action recognition or monitoring are handled by other layers, and the underlying reasoning tasks are not implemented as explicit symbolic rules in the knowledge base.

The experiments we have conducted are also likely to have too simplistic semantics to let complex reasoning needs emerge. Scenarios with more complex semantics would be desirable to better stress the expressiveness and inference abilities provided by Description Logics.

Is reasoning at the knowledge level immature or even superfluous, then? Not so: hundreds of trivial (from a human point of view) inferences are continuously produced by the system (translating inheritance relations, domain/range constraints, transitivity, etc.) and encode a large amount of common-sense knowledge that can not be otherwise conveniently asserted. These trivial inferences are all the more important that an expressive knowledge representation language is used. Since a language like OWL allows it to directly represent high-level concepts like partitions, cardinality restrictions, properties' ranges and domains, it leads to a more implicit description of the available knowledge. This in turn pushes for a lot of "trivial" reasoning to make the knowledge pool explicit, and hence largely reusable by the deliberative subsystems. With the progress in the understanding of the relations between expressiveness and (tractable) satisfiability, along with the progress of reasoners, more and more of the assertions do not need to be explicit anymore, and consequently are delegated to the reasoner.

We think that common-sense encoding is likely to remain the main application of reasoning in our architecture, where reasoning related to decision making mostly happens outside the knowledge representation system.

\section{The Cognitician view: palpable knowledge and semantic thinking}

The original motivation to introduce explicit knowledge management in our architecture was to transform the knowledge in the robot from some ubiquitous, pervasive, multimodal and, most importantly, mostly undefined feature of the system into an observable, quantifiable, manipulable resource: a palpable feature of the system.

This transformation, both from the technical point of view (the ORO server, the ontologies, the bindings, etc.) and as a more subtle change in the practises related to the development of robotic components, is probably the main outcome of this work.

Knowledge is not an abstract concept anymore: it is a set of statements, in most cases directly intelligible to the developers, stored in one place. We can export them, monitor them, review them, question them.

Communication between the robot's modules is now conceived in terms of what are the semantics of the information flows, instead of a simple compatibility of interfaces. When defining the frontiers of a robotic component, we do not think anymore only in terms of is the interface complete and selfcontained, but also in terms of is the semantic complete and consistent?. This allows a deeper, more correct modularity: two modules that share the same, well-defined semantic can be confidently exchanged. When we remove or disable a component (the dialogue processing, the geometric reasoning, ...), we know precisely what knowledge will not be available anymore.

We call this new property of our robot, that allows for both qualitative and quantitative analysis of the beliefs, its cognitive observability.

It is somewhat related to the idea of cognitive penetrability introduced by Pylyshyn [22] in 1989, in the context of the study of possible strong equivalences between computational models and the psychological reality:

[One of the criterion] relies on the assumption that we can identify certain clear cases of phenomenon that should be accounted for at the knowledge level, that is, in terms of the representations alone, rather than in terms of properties of the cognitive architecture. Phenomena that depend in a rational way on subjects' goals, beliefs, and utilities are a case in point. For example in psychophysics we 
assume that if a measure (such as a threshold) changes systematically as we change the payoffs (that is, the relative cost of errors of commission and of omission), then the explanation of that change must be given at the knowledge level in terms of decision theory - rather than in terms of properties of sensors or other mechanisms that are part of the architecture. In general showing that certain empirical phenomena are sensitive to goals and beliefs (or what I call cognitively penetrable) is prima facie evidence that they should not be attributed to properties of the architecture.

The introduction of an explicit knowledge level in our architecture makes it possible to effectively assess the cognitive penetrability of the whole robot behaviours (this is however not new, and traditional BDI architectures would also make this claim).

Even more, this architecture also contributes to bring closer robotics and cognitive psychology: it provides clear entry points to implement some classical psychology tests to robots, be it related to perspective taking, to language understanding, to a theory of mind (such as the False-Beliefs experiment), etc.

\section{CONCLUSION}

This article does not claim to provide an exhaustive assessment of the strengths and weaknesses of explicit knowledge management in a large robotic architecture. Most of the "lessons" that we have "learned" are not new, and the various scientific communities (task planning, architecture control, NLP, geometric reasoning, social psychology...) that are related to human-robot interaction and robotic cognition already know them.

One of the difficult challenges of cognitive robotics, however, is to overcome the relative isolation of each of these communities to build an autonomous embodied agent, able to interact with humans. This contribution modestly offers a critical look at one instance of such a "real-world" robotic architecture, that tackles several of the facets of cognitive robotics.

If we had a single "lesson" to retain, it would likely be that one: shifting from a robotic design based on modules and APIs to a system based on modules and human-level semantic interfaces has been the main facilitator both for glueing together heterogeneous deliberative modules in a meaningful way, and quickly developing prototypes for new upper-cognition abilities, like natural dialogue and theory of mind.

\section{ACKNOWLEDGMENT}

This work has been supported by EU FP7 "SAPHARI" under grant agreement no. ICT-287513.

\section{REFERENCES}

[1] J. Mainprice, E. A. Sisbot, L. Jaillet, J. Cortes, R. Alami, and T. Simeon, "Planning human-aware motions using a sampling-based costmap planner," in IEEE International Conference on Robotics and Automation, 2011.
[2] J. Mainprice, M. Gharbi, T. Siméon, and R. Alami, "Sharing effort in planning human-robot handover tasks," in RO-MAN, 2012.

[3] A. Pandey, M. Ali, M. Warnier, and R. Alami, "Towards multi-state visuo-spatial reasoning based proactive human-robot interaction," in Proceedings of the 15th International Conference on Advanced Robotics. IEEE, 2011, pp. 143-149.

[4] S. Lemaignan, R. Ros, L. Mösenlechner, R. Alami, and M. Beetz, "ORO, a knowledge management platform for cognitive architectures in robotics," in IEEE/RSJ International Conference on Intelligent Robots and Systems, 2010.

[5] R. Ros, S. Lemaignan, E. A. Sisbot, R. Alami, J. Steinwender, K. Hamann, and F. Warneken, "Which one? grounding the referent based on efficient human-robot interaction," in 19th IEEE International Symposium in Robot and Human Interactive Communication, 2010.

[6] S. Lemaignan, R. Ros, E. A. Sisbot, R. Alami, and M. Beetz, "Grounding the interaction: Anchoring situated discourse in everyday human-robot interaction," International Journal of Social Robotics, pp. 1-19, 2011.

[7] M. Warnier, J. Guitton, S. Lemaignan, and R. Alami, "When the robot puts itself in your shoes. Explicit geometric management of position beliefs." in Proceedings of the 21st IEEE International Symposium on Robot and Human Interactive Communication, 2012.

[8] R. Alami, M. Warnier, J. Guitton, S. Lemaignan, and E. A. Sisbot, "When the robot considers the human..." in Proceedings of the 15th International Symposium on Robotics Research, 2011, to appear.

[9] E. Sisbot, R. Ros, and R. Alami, "Situation assessment for humanrobot interaction," in 20th IEEE International Symposium in Robot and Human Interactive Communication, 2011.

[10] S. Alili, M. Warnier, M. Ali, and R. Alami, "Planning and planexecution for human-robot cooperative task achievement," in 19th International Conference on Automated Planning and Scheduling, 2009.

[11] M. Woolridge, Multiagent Systems. A Modern Approach to Distributed Artificial Intelligence. Massachusetts Institute of Technology, 1999, ch. Intelligent Agents, pp. 27-78.

[12] S. Lemaignan, Grounding the Interaction: Knowledge Management for Interactive Robots, 2012, ch. The Knowledge API, pp. 161-174.

[13] M. Tenorth and M. Beetz, "KNOwRoB - knowledge processing for autonomous personal robots," in IEEE/RSJ International Conference on Intelligent Robots and Systems, 2009.

[14] G. Lim, I. Suh, and H. Suh, "Ontology-based unified robot knowledge for service robots in indoor environments," IEEE Transactions on Systems, Man and Cybernetics, pp. 1-18, 2011.

[15] X. Chen, J. Ji, J. Jiang, G. Jin, F. Wang, and J. Xie, "Developing high-level cognitive functions for service robots," in Proceedings of the 9th International Conference on Autonomous Agents and Multiagent Systems, ser. AAMAS '10. Richland, SC: International Foundation for Autonomous Agents and Multiagent Systems, 2010, pp. 989-996.

[16] E. Erdem, E. Aker, and V. Patoglu, "Answer set programming for collaborative housekeeping robotics: representation, reasoning, and execution," Intelligent Service Robotics, vol. 5, no. 4, pp. 275-291, 2012.

[17] C. Breazeal, M. Berlin, A. Brooks, J. Gray, and A. Thomaz, "Using perspective taking to learn from ambiguous demonstrations," Robotics and Autonomous Systems, pp. 385-393, 2006.

[18] R. Ros, E. A. Sisbot, R. Alami, J. Steinwender, K. Hamann, and F. Warneken, "Solving ambiguities with perspective taking," in 5th ACM/IEEE International Conference on Human-Robot Interaction, 2010.

[19] S. Lemaignan, "Grounding the interaction: Knowledge management for interactive robots," Ph.D. dissertation, CNRS - Laboratoire d'Analyse et d'Architecture des Systèmes, Technische Universität München Intelligent Autonomous Systems lab, 2012.

[20] D. Premack and G. Woodruff, "Does the chimpanzee have a theory of mind?" Behavioral and Brain sciences, vol. 1, no. 4, pp. 515-526, 1978 .

[21] A. Leslie, "Theory of mind as a mechanism of selective attention," The new cognitive neurosciences, pp. 1235-1247, 2000.

[22] Z. Pylyshyn, Foundations of cognitive science. Cambridge: A Braddford Book, The MIT Press, 1989, ch. Computing in cognitive science, pp. 51-91. 